\title{
Multi-Objective Path Optimization for Critical Infrastructure Links with Consideration to Seismic Resilience
}

\author{
Zengfu Wang \\ School of Automation, Northwestern Polytechnical University, Xi'an, China \\ Qing Wang \& Moshe Zukerman \\ Department of Electronic Engineering, City University of Hong Kong, Hong Kong, China
}

Jun Guo

School of Computer Science and Network Security, Dongguan University of Technology, Dongguan, China

Yu Wang *

Department of Architecture and Civil Engineering, City University of Hong Kong, Hong Kong, China

Gang Wang

Department of Civil and Environmental Engineering, Hong Kong University of Science and Technology, Hong Kong, China

Jun Yang

Department of Civil Engineering, The University of Hong Kong, Hong Kong, China

$\&$

Bill Moran

School of Engineering, RMIT University, Melbourne, Australia

Abstract: We study the generic problem of path optimization for a critical infrastructure link between two locations on the surface of the Earth in the vicinity of earthquakeprone areas. The problem has two (conflicting) objective functions, one for minimizing the construction cost of the link and the other for minimizing the number of potential repairs along the link in the wake of earthquakes. In our model, the Earth's surface is approximated by a triangulated manifold, and ground motion intensity data are used to provide a measure of repair rate. We approach the multi-objective variational problem by first converting it into a single objective variational problem using the weighted sum method. Then, we show that the problem can be further transformed into an Eikonal equation and solved by a computationally efficient

\footnotetext{
${ }^{*}$ To whom correspondence should be addressed. E-mail:
} yuwang@cityu.edu.hk algorithm based on the Fast Marching Method. Extensive simulations are performed on real-world three-dimensional geographical data, from which we obtain Pareto optimal solutions that provide insight and guidance to design tradeoffs between cost effectiveness and seismic resilience.

\section{INTRODUCTION}

Critical infrastructures such as electricity, oil, gas, telecommunications, transportation and water are essential to the functioning of modern economies and societies. As the world is increasingly interconnected, long-haul transregional, -national, or -continental links are playing a crucial role in transporting critical resources and information from one location to another. For example, it is known that submarine telecommunications cables carry over $95 \%$ of the global voice and data traffic (Carter et al., 2009). Russian gas that

This is the author manuscript accepted for publication and has undergone full peer review but has not been through the copyediting, typesetting, pagination and proofreading process, which may lead to differences between this version and the Version of Record. Please cite this article as doi: $10.1111 /$ mice.12287

This article is protected by copyright. All rights reserved. 
is delivered through the trans-European pipeline accounts for over a quarter of the total European consumption (Cobanli, 2014). Such critical infrastructure links are vulnerable to disasters (Neumayer et al., 2011) and, if broken, can have severe social and economic consequences.

Among various natural disasters, earthquakes often cause the most catastrophic effects. For example, in 1987, the Ecuador earthquake resulted in the damage of nearly $70 \mathrm{~km}$ of the Trans-Ecuadorian oil pipeline. Loss of the pipeline deprived Ecuador of $60 \%$ of its export revenue, and it took five months to reconstruct the pipeline (Schuster, 1991). In 2006, the Hengchun/Taiwan earthquake damaged eight submarine cables with a total of 18 cuts. As a result, Internet services in Asia were severely disrupted for several weeks, affecting many Asian countries (Qiu, 2011). It was estimated that, for a well-developed economy that is largely reliant on the Internet, one week of Internet blackout can cause losses of over 1\% of annual GDP (mi2g, 2005; Dübendorfer, 2005). These events signify the impacts of earthquake hazards and the importance of enhancing the seismic resilience of critical infrastructure links (Cao et al., 2013; Cao, 2015; Cao et al., 2016).

In this paper, we study the generic problem of path optimization for a critical infrastructure link between two locations on the surface of the Earth that crosses an earthquake-prone area. The focus is on infrastructure links, such as undersea cables and long-haul oil/gas/water pipelines, where surface distance is a reasonable measure of the length of a link. For such a practically important problem, where the topography of the landform between the two locations is given, we are not aware of any theoretically sound approach proposed in the literature that takes into consideration both cost effectiveness and seismic resilience. To address this gap, we formulate the problem as a multi-objective variational problem where we aim to find the set of Pareto optimal paths for the infrastructure link with two objective functions.

- The first objective is to minimize the cost associated with the construction of the infrastructure link. Connecting the two locations by a geodesic, i.e., the route with the shortest surface distance, may minimize the construction cost but can increase the risk of damage or break in the event of an earthquake if the route is close to the hazard.

- The second objective is to minimize the number of potential failures (hence repairs) along the infrastructure link in the wake of earthquakes, which may serve as an index of the cost associated with the loss and reconstruction of the link in the event of failures.

Note that the second objective in our context is related to the notion of seismic resilience (Bruneau et al., 2003). Ac- cording to Bruneau et al. (2003), two key measures of resilience in general and seismic resilience in particular are "reduced failure probabilities" and "reduced time to recovery”. Røstum (2000); Fragiadakis and Christodoulou (2014) have shown that the larger the number of potential repairs, the greater is the failure probability of a link. In this paper we will reduce both the failure probability and the time to recovery by minimizing the total number of potential repairs for a given budget, and therefore improve seismic resilience.

Our model is built on the state of the art in geographic information systems (GIS) for terrain approximation (Chang, 2013) and, ground movement based risk evaluation, the latest development in civil engineering for seismic risk assessment of critical infrastructure links (Jeon and O'Rourke, 2005; Fragiadakis and Christodoulou, 2014). Specifically, the model considers triangulated manifolds for representing the surface of the Earth, and ground motion intensity measures for estimating the link repair rate in the event of an earthquake since the correlations between them can be found statistically from past earthquakes (Jeon and O'Rourke, 2005; Wang and O'Rourke, 2008; Pineda-Porras and Najafi, 2010; Fragiadakis and Christodoulou, 2014). Based on this model, we approach the multi-objective variational problem by first converting it into a single objective variational problem using the weighted sum method (Miettinen, 1999). Then, we show that the problem can be further transformed into an Eikonal equation and solved by a computationally efficient algorithm based on the well-established Fast Marching Method (FMM) (Kimmel and Sethian, 1998; Sethian, 1999a). This enables us to obtain Pareto optimal solutions that provide flexibility in path optimization for a critical infrastructure link, taking into consideration the fundamental tradeoff between cost effectiveness and seismic resilience. In summary, our novelty here is twofold:

1. For the first time, we apply a repair rate model based on ground motion intensities, a landform model based on a triangulated manifold, and an additive construction cost model for link construction optimization.

2. For the first time, we apply the FMM algorithm to the multi-objective variational optimization problem of minimizing construction cost while minimizing total number of repairs.

The rest of the paper is organized as follows. In Section 2, we discuss the related work. In Section 3, we discuss the issues in constructing a link between two nodes and the rationale for a multi-objective optimization approach. In Section 4, we describe the models. In Section 5, we provide details of the problem formulation and solution. Simulation results are presented in Section 6. Finally, we draw conclusions in Section 7.

This article is protected by copyright. All rights reserved. 


\section{RELATED WORK}

Much work has been done on understanding the damage of infrastructure links by past earthquakes. The work of Chen et al. (2002) investigated the damage patterns of natural gas and water pipelines in the 1999 Chi-Chi earthquake, and conducted statistical analysis to understand the correlation between repair rates and seismic parameters. Considering the same 1999 Chi-Chi earthquake, Hwang et al. (2004) investigated damage to natural gas pipelines due to ground shaking effects, and performed regression analyses of pipe repair rates to derive seismic vulnerability functions based on pipe repair data and recorded strong motion data. The work of Liu (2009) summarized main factors that impact submarine cables based on their performance in three past earthquakes, including the 2006 Hengchun earthquake, the 2004 Sumatra earthquake and the 1929 Grand Banks earthquake. The work of Carter et al. (2014) investigated the effect of damaging submarine flows on submarine cables in the 2006 Pingtung earthquake, and presented insights regarding the causes, frequency, and behavior of submarine flows. The work of Kobayashi (2014) reported the experience of infrastructure damage caused by the 2011 Tohoku earthquake.

Through modeling and analyzing the vulnerability, researchers have also worked on evaluation of potential damage to current infrastructure links by earthquakes. Lanzano et al. (2013) analyzed the interaction of earthquakes with natural gas pipelines in terms of the likelihood of the loss of containment with respect to peak ground velocity (PGV). Esposito et al. (2015) analyzed the vulnerability of gas networks via fragility curves and evaluated their seismic performance via computer-aided simulation. Wang and O'Rourke (2008); Wang and Au (2009) proposed methods to identify critical links of water supply with a relatively large damage probability under an earthquake. Adachi and Ellingwood (2009) provided an evaluation of the serviceability of the municipal water distribution system in Shelby County, Tennessee considering spatial correlation in seismic intensity and demand. A case study for a town in suburb of Algiers has been presented in Zohra et al. (2012) for a proposed method based on the identification of parameters to assess the seismic vulnerability of water pipeline network. Cavalieri et al. (2014) presented a comparison of five seismic performance assessment models for power networks.

The work mentioned above focused on modeling, analysis or evaluation of potential damages and vulnerability for a given infrastructure link system, but not on the path optimization for a critical infrastructure link which is the problem considered in this paper. Although the former can provide insights and support for the latter, however, they are much more distinct in methodologies.
A closely related problem in civil and infrastructure engineering is pipeline route selection. Traditionally, in practice, the route selection procedures of critical infrastructure links have been achieved manually based on expert experience (Burnett et al., 2013). In this approach, engineers draw several relatively reasonable paths on a large-scale topographical map using available data, such as maps and aerial photographs, of the region of interest. Then, to verify the availability and rationality of the selected paths (Iqbal et al., 2006), a survey is conducted along them. If the survey reveals constraints or obstacles that cannot be eliminated or removed, new paths have to be chosen manually and verified by survey (Berry et al., 2004; Holmes and Squire, 2012). The final path of the link is determined by detailed analyses and comparisons. The traditional manual approach depends on expert judgment and subjective analysis, which may be far from optimal and especially when the decision space is large and complex (Holmes and Squire, 2012).

State-of-the-art approaches are in general computer-assisted heuristics utilizing GIS technology (Dey and Ogunlana, 1999; Yildirim et al., 2007; Balogun et al., 2012; Macharia, 2014). Specifically, they are based on weighting factors considered to be affecting the route and then applying rasterbased path analysis to find the least accumulative cost path using the (discrete) Dijkstra's shortest path algorithm (Chang, 2013). However, the effects of earthquakes were not considered by these publications. A similar approach was used by Zhao et al. (2017) for cable route selection considering cost minimization and earthquake survivability. A major limitation of the raster-based path analysis is that a path is restricted to use either a lateral link or a diagonal link when moving from one cell to its adjacent cells. For a given grid graph, even with very small grid/cell size, this is an inherent limitation (Sethian, 1999a). From a practical point of view, although using a finer grid graph to model the configuration space of the path analysis problem would reduce the shortcoming of the raster-based method, it would also dramatically increase the computational expense. Our approach in this paper suits a broader class of critical infrastructure links including undersea cables and uses a theoretically sound methodology with guaranteed optimality within the approximations and the limitations of the available data and link construction cost and failure models.

The use of multi-objective (or multi-criteria) optimization in infrastructure design is not new (Sarma and Adeli, 2000b; Sirca Jr and Adeli, 2005; Pérez et al., 2015). There also exist studies on the topic of cost-effectiveness or cost-benefit analysis, e.g., for mitigation (Rose et al., 2007), restoration (Bocchini and Frangopol, 2012) and health care intervention (Neumann et al., 2014). These and other existing publications on multi-objective optimization in the general area of infrastructure design or cost-effectiveness analysis

This article is protected by copyright. All rights reserved. 
did not consider the critical infrastructure link path optimization problem developed in this paper. In Cao et al. (2013); Cao (2015); Cao et al. (2016), there has been an attempt to apply multi-objective optimization to path planning of a telecommunication cable. However, in these publications, it is assumed that the topology lies on a two-dimensional plane. We address the path optimization problem for infrastructure links based on a more accurate model that represents the surface of the Earth as a two-dimensional manifold in threedimensional space; that is, we account for topography and terrain.

\section{THE MULTI-OBJECTIVE OPTIMIZATION APPROACH}

As discussed in the Introduction, the optimization of the path of a link between two locations in an earthquake prone region is based on multiple objectives. In particular, we consider the following two objective functions. The first is the laying cost (applicable to e.g. a telecommunication cable), or construction cost (for e.g. an oil pipeline). For brevity, thereafter, we will use the term construction cost to refer to both laying and construction cost. The second objective function is an index associated with the estimation of future number of repairs (or failures) of the link in a given time period (e.g. 100 years). While the first objective is about cost incurred during construction, the second objective is about cost incurred in the (potentially, long term) future.

\subsection{Why multi-objective optimization?}

There are various factors associated with estimation of the first objective, namely, the construction cost. The length of the link is clearly a factor here, but it is not the only factor as the construction cost can vary from one location to another based on ground/soil condition, requirement for security arrangements, licensing and various other factors. The reason that we need to address the problem as a multi-objective optimization is the second objective function.

While the dollar value of the first objective is relatively clear, it is not so simple to assign a dollar value to potential link failures, mainly because different stakeholders have significantly different views of the cost of link failures. While for a telecom cable owner, cable breaks incur cost associated with the repair needed minus any insurance payment received, for an insurance company the cost consequence may be higher, and for the society, government or public the cost of cable failures can be much higher, as one week of Internet shutdown has been estimated at $1.2 \%$ of annual GDP (mi2g, 2005; Dübendorfer, 2005), which means billions of dollars. In addition, failure of infrastructure links can lead to loss of lives in various cases of natural disasters. Given the multiplicity of stakeholders with different exchange rates between link failure risks and dollar values, it is appropriate to use a methodology based on multi-objective optimization that leads to a set of Pareto optimal solutions. Such optimal solutions provide for a given budget for construction cost, the planned path that minimizes the risk (as measured by predicted number of repairs), and for each given predicted number of repairs, the planned path that minimizes the construction cost.

\subsection{The second objective}

While the choice of the first objective is relatively straightforward the choice of the second objective of the predicted number of repairs requires some discussion. Larger predicted number of repairs (failures) indicates both potential costs of repairs, as well as link downtime that may have significant societal cost. As an illustration, after the 2006 Taiwan Earthquake, eight submarine cable systems were found to be damaged with a total of 18 cable cuts (Qiu, 2011). The repair for each cable cut was expected to require around seven days (LaPerrière, 2007). Although some repairs can be done in parallel, it still took over a month to achieve full restoration of connectivity following the Taiwan earthquake.

Accordingly, we adopt the view that a reasonable index to represent the level of damage caused by an earthquake is the total number of repairs (or failures) of the link. To estimate the number of repairs, we rely on data of ground motion in the past during a certain period of time, or simulations based on given geological knowledge. We are, in fact, using past earthquake data to predict seismic events in the future. Nevertheless, this is considered reasonable, as the geology does not change significantly over the service life of the link.

Since the relevant period of time the ground motion data has been measured (or simulated) applies to all points in the map equally, and since the data is based on the past, we henceforth use the abbreviated term of total number of repairs without mentioning the period of time and the fact that it is "potential". This is our second objective to be optimized.

To calculate total number of repairs for a link, we will use the term repair rate (Jeon and O'Rourke, 2005; Wang and O'Rourke, 2008; Esposito, 2011; Fragiadakis and Christodoulou, 2014; Cimellaro et al., 2014) to indicate the predicted number of repairs per unit length of the link over a fixed time period into the future. An alternative term, less used in the earthquake literature is failure rate. In addition, for a specific link, the repair rate varies for different points on the link and depends on various factors as well, such as the geology, link material and ground/soil conditions. In another context considering earthquakes effects, the repair rate

This article is protected by copyright. All rights reserved. 
has been widely used to assess reliability of water supply networks (Jeon and O'Rourke, 2005; Wang and O'Rourke, 2008; Fragiadakis and Christodoulou, 2014), and to analyze the risk to gas distribution networks (Esposito, 2011; Cimellaro et al., 2014).

To estimate the repair rate which we use for estimating the total number of repairs of a link, we rely on data of ground motion in the past during a certain period of time, or simulations based on given geological knowledge. As in Section 4, we also take advantage of the extensive work of the United States Geological Survey (USGS) analysts that develop models for the potential effects of future earthquakes.

The total number of repairs (and repair rate) indicates both the expected time period between the seismic events that will result in repairs and their probability of occurrence. The higher the probability of occurrence and intensity of seismic events, the larger the ground motion intensity and therefore the larger the repair rate.

In this paper, we consider two objectives-construction cost and number of potential repairs. Other objectives are easily integrated into our approach if they can be computed as an integral of some quantity along the path. Effectively this means the objectives are local and additive across multiple path segments.

\section{MODELLING}

In this section, we describe the models we introduce for the landforms, construction cost, and the potential required repairs.

\subsection{Landform model}

We approximate the region of the Earth's surface (including the sea bed) under consideration as a closed, connected (Greenspan, 2000), two-dimensional manifold in three-dimensional Euclidean space $\mathbb{R}^{3}$, uniquely represented by a continuous, single-valued function $z=\xi(x, y)$, where $z$ is the elevation and $x$ and $y$ are the Cartesian coordinates (Florinsky, 2012). In particular, caves, grottos, tunnels etc. are ignored.

As information about the landforms is always available in a quantized form (discrete grid), we use a triangulated piecewise-linear two-dimensional manifold $\mathbb{M}$ to approximate the Earth's landforms. Such triangulated manifold models are widely used to represent topography and terrain in GIS and other related fields, as they makes it easier than other available models (e.g. the regular grid model) to consider rough surfaces and to accommodate irregularly spaced elevation data (Peucker et al., 1978; Lee, 1991). We further
Table 1: Key Notations

\begin{tabular}{cc}
\hline $\mathbb{R}^{n}$ & $n$-dimensional Euclidean space \\
$\mathbb{M}$ & triangulated piecewise-linear two-dimensional \\
& manifold in $\mathbb{R}^{3}$ \\
$(x, y, z), z=\xi(x, y)$ & coordinates of a point in $\mathbb{M}$ \\
$h(x, y, z)$ & construction cost at point $(x, y, z)$ \\
$A, B$ & two nodes to be connected by a link in $\mathbb{M}$ \\
$X_{A}, X_{B}$ & coordinates of $A, B$ \\
$\gamma$ & a link, a path or a curve representing the link \\
$\mathbb{H}(\gamma)$ & the construction cost of the link $\gamma$ \\
$S, X, X_{S}$ & points in $\mathbb{M}$ \\
$g(X)$ & repair rate at location $X$ \\
$\mathbb{G}(\gamma)$ & total number of repairs of the link $\gamma$ \\
$l(\gamma)$ & length of the link $\gamma$ \\
$f(X(s))$ & weighted summation of construction cost \\
$\phi(S)$ & and repair rate at location $X(s)$ \\
$\nabla$ & minimal cumulative cost to travel \\
$\|\cdot\|$ & from one point to a point $S$ \\
$\triangle V V_{1} V_{2}$ & gradient operator \\
$\bar{\phi}(V)$ & 2-norm \\
$\Gamma$ & a triangle in $\mathbb{M}$ \\
$N$ & PDid point set of $\mathbb{M}$ \\
$m$ & Palue at $V$ calculated by numerical method \\
$r$ & total number of discretized grid points of $\mathbb{M}$ \\
$v$ & earthquake magnitude \\
$c$ & mearthquake magnitude $m$ \\
$\mu$ & epicenter distance from a site to a fault line source \\
$f_{M}(m)$ & PGV \\
$f_{R}(r), F_{R}(r)$ &
\end{tabular}

assume that the triangulated manifold model is complete; that is, it is a connected triangulated manifold surface in $\mathbb{R}^{3}$ that consists of faces, edges and vertices and satisfies the following conditions (Martínez et al., 2005).

- There are no isolated vertices.

- Each edge belongs to exactly one triangle or is shared by just two triangles. Any two triangles intersect in a common vertex or edge, or not at all.

- Any two points on the surface, are connected by a path (possible through the middle of a triangle) on the surface connecting the two points.

These conditions do not pose significant modeling limitations because areas that do not satisfy these conditions, such as cliff faces, will be avoided by the link in any case. The particular details of how to address such areas in the model will be discussed below.

\subsection{Construction cost model}

As mentioned in Section 3, the construction cost is affected by various factors and varies from one location to another. For $(x, y, z) \in \mathbb{M}$, we define a function $h(x, y, z)$ to represent

This article is protected by copyright. All rights reserved. 
the link construction cost at point $(x, y, z)$, where $z=\xi(x, y)$. This function gives the path planner the flexibility to consider different construction cost for different locations. For example, there are many areas that links (submarine telecommunications cables) must avoid, or require high construction costs (Yung, 2011). They include:

- areas that are of high ecological value (e.g. coral communities)

- areas where special and costly licenses are required

- incompatible seabed (e.g. rocky areas)

- big mountains and even man-made obstructions

- any other areas that must be avoided

Setting appropriately high values to the function $h(x, y, z), z=$ $\xi(x, y)$ will enable avoidance of such areas, or at least imposition of a high construction cost. In areas where the construction cost of the link is only its length, we set $h(x, y, z)$ equal to a constant value, e.g., $h(x, y, z)=1$, where $z=\xi(x, y)$.

Let node $A$ and node $B$ be two fixed points with coordinates $X_{A}$ and $X_{B}$ in $\mathbb{M}$, that have to be connected by a link, defined as a (Lipschitz continuous (Eriksson et al., 2013)) curve $\gamma$ in $\mathbb{M}$ that connects the points $A$ and $B$. The assumption of Lipschitz continuity is necessary to guarantee the existence of the solution of the Eikonal equation introduced in Section 5. Fortunately, in practice, this condition has negligible effect on the accuracy of our solution. Lipschitz continuity requires that the slope of the secant line joining two different points on the link is always bounded by a finite number, and is weaker in our context than differentiability with continuous derivative. In other words, the link cannot be infinitely steep at one point. This in practice implies a very steep cliff (exactly vertical) that the link must avoid in any case. Avoidance of such areas is part of our optimization as discussed above. Let $\mathbb{H}(\gamma)$ be the construction cost of the link $\gamma$. We assume:

- The construction cost $\mathbb{H}(\gamma)$ of the link $\gamma$ is quantified in terms of the cost per unit length at every point on the link, and is location dependent.

- For any particular point on the link, $S$, the construction cost per (arbitrarily) small length $d s, d \mathbb{H}(\gamma)$, is calculated as the product of the construction cost $h\left(X_{S}\right)$ and length $d s$, i.e., $h\left(X_{S}\right) d s$. Here we use capital letters (e.g. $S, X, X_{S}, A$, and $B$ ) to denote points, but we use small letters (e.g., $x, y, z)$ to denote the actual coordinates.

Then the construction cost of the link $\gamma$ is the integral of the construction cost at each point along the path of the link. That is,

$$
\mathbb{H}(\gamma)=\int_{\gamma} h(X) d s
$$

where $h(X) \in \mathbb{R}_{+}^{1}$ is the construction cost at location $X$.

Note that construction cost evaluation is a complicated process. Real costs depend on the specific application to be solved. Our model above can handle many constraints which can be modeled by an additive construction cost. However, it is unable to consider constraints with non-additive property (e.g. bulk discount). More realistic infrastructure construction cost models and analysis can be found in Adeli and $\mathrm{Wu}$ (1998); Adeli and Sarma (2006); Rui et al. (2011).

\subsection{Link repair model}

Now we discuss the correlation of ground movements resulting from earthquakes with the repair rate.

Since the link is laid on the surface of the Earth, the repair rate $g(X)$ is defined on the surface introduced previously, and therefore as a function of the coordinates $x$ and $y: g(X)=$ $g(x, y, z), z=\xi(x, y)$. Typically, after an earthquake event, the area affected can be subdivided into many cells, and in each cell, repair rate of a link in the cell is determined by dividing the number of repairs by the length of the link in the cell.

Some publicly available information on the repair rate and its correlation with ground movement can be found in the context of water and gas pipelines (Jeon and O'Rourke, 2005; Wang and O'Rourke, 2008; Fragiadakis and Christodoulou, 2014). Many ground motion parameters have been used for relating repair rate with seismic intensity (Pineda-Porras and Najafi, 2010). In this paper, PGV is adopted to derive the repair rate since a significant correlation has been found between the two (O'Rourke et al., 1998; Toprak, 1998; Toprak and Taskin, 2007) and PGV is widely used for deriving repair rate in the literature (Alliance, 2001; Jeon and O'Rourke, 2005; Pineda-Porras and Najafi, 2010). Note that Newmark (1968) developed a theoretical model for seismic wave interaction with pipelines and showed that the seismic wave induced strain along the pipeline is proportional to the ground velocity. An example of PGV map of Contiguous United States, derived based on Peak Ground Acceleration (PGA) (http: //www .usgs.gov/) data provided by USGS, is shown in Fig. 1. For specific details on how we convert data from PGA to PGV see Section 6 below. In Fig. 1, different colors represent different levels of PGV. We can read the value of PGV (in $\mathrm{cm} / \mathrm{s}$ ) for a site from the color bar on the right of Fig. 1. The gradual change of the color of the color bar, which is from blue to red, corresponds to increases of the PGV from the minimum value to the maximum value.

Note that the application of our method is not limited to PGV and other information on ground motion can be used to es-

This article is protected by copyright. All rights reserved. 


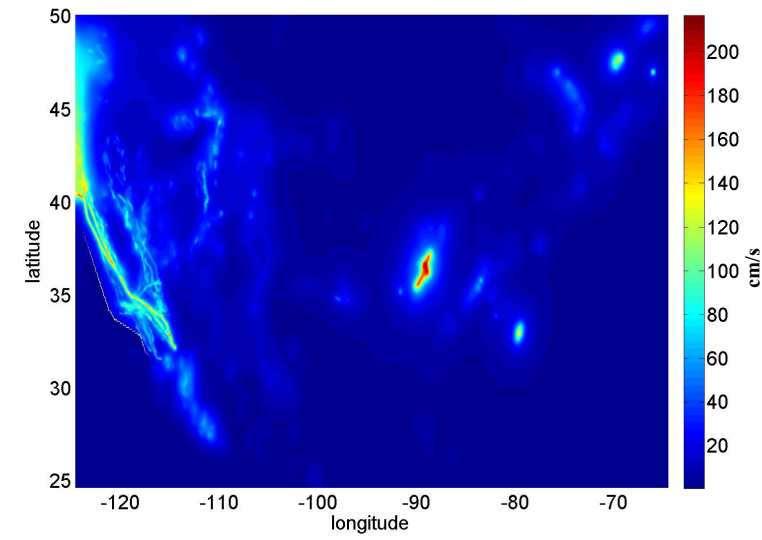

Figure 1: The shaded surface map of PGV of Contiguous United States $(\mathrm{cm} / \mathrm{s})$. Data is provided by USGS.

timate the repair rate. It is apparent that the more accurate the estimation of repair rate is, the more reliable are the path planning results.

As we mentioned in the description of modeling of construction cost, assigning high positive repair rate values to the function $g(x, y, z), z=\xi(x, y)$ will force the link to avoid high risk areas. For example, as discussed by Yung (2011), the following are high risk areas for submarine telecommunications cables:

- areas with wind or underwater turbines

- marine vessel fairways

- marine borrow area (e.g. gazetted dredging and sediment disposal area and sand deposit area)

- anchorage areas and fishery areas.

Other adjustments can be made to the repair rate function to consider particular link attributes.

Let $\mathbb{G}(\gamma)$ be the total number of repairs of the link $\gamma$. The assumptions we made previously for the construction cost of the link $\mathbb{H}(\gamma)$, apply also for the total number of repairs; namely, we assume,

- The total number of repairs $\mathbb{G}(\gamma)$ of the link $\gamma$ is quantified in terms of the repair rate at every point on the link, and is location dependent.

- For any particular point on the link, $S$, the number of repairs per (arbitrarily) small length $d s, d \mathbb{G}(\gamma)$, is calculated as the product of the repair rate $g\left(X_{S}\right)$ and length $d s$, i.e., $g\left(X_{S}\right) d s$.

Then the total number of repairs of the link $\gamma$ is the integral of the repair rate at each point along the path of the link. That is,

$$
\mathbb{G}(\gamma)=\int_{\gamma} g(X) d s,
$$

where $g(X) \in \mathbb{R}_{+}^{1}$ is the repair rate at location $X$.

Note that many influencing factors may affect failures of critical infrastructure links although mainly earthquake hazard is considered in this paper. Our methodology can be extended to include other factors, for example landslides, if the corresponding repair rate models can be provided. Equivalently, as discussed in our cost model description, our repair rate model can include influencing factors provided they are local and additive in nature.

\section{PROBLEM FORMULATION AND SOLUTION}

In this section, we first provide a rigorous formulation of our multi-objective optimization problem with objective functions - the construction cost and the total number of repairs, based on the models of landforms, construction cost, and the potential required repairs. Then, we describe in detail the algorithm that obtains a set of path planning alternatives, each of which achieves optimal construction cost for each value of required repairs, and the least number of required repairs for a given budget. These set of alternatives form the so called Pareto optimal set. We note that our use of terms "optimal" and "least" here represent the best we can do given a grid size of the topographic data and any discrepancy that may be introduced by the manifold $\mathbb{M}$ relative to the real landform. As this grid size is reduced, potentially, better solutions may be obtained. The advantage of this approach is that it provides the true optimal results given the resolution level, the data, the construction cost model and the failure model.

First, considering the functions of construction cost and repair rate defined on the manifold $\mathbb{M}$ that represents the real landform of the area under consideration, we formulate path planning as a multi-objective variational optimization problem, called Problem 1. In this optimization problem, the objective functions are (1) the total construction cost, and (2) the total number of repairs accumulated for any given path. The values of these objective functions are obtained by line integrals over each path. Then we transform our multiobjective variational optimization problem to a single combined objective problem, called Problem 2, where at each point on the manifold, a single function based on a fixed weighted average of the construction cost and the repair rate is considered. According to Bector and Husain (1992), Problem 2 provides a Pareto optimal set as the weights are varied in the calculation of the single combined objective function.

Problem 2 is transformed to the so-called Eikonal equation; a non-linear partial differential equation (PDE) encountered in problems of wave propagation. Intuitively, given the Start Point $A$, a propagated wave front from this point represents 
a set of points that achieve the same value for the minimal line integral from Start Point $A$ (to the point of the wave front) of the single combined objective function. A solution of the Eikonal equation provides fronts of the waves where the front most distant from Start Point $A$ includes End Point $B$. Then the desired optimal path is obtained be moving from End Point $B$ towards Start Point $A$ through all the wave fronts by always choosing the steepest decent.

Recall that the proposed solution of Problem 2 requires a continuous manifold $\mathbb{M}$, and that we only have the discretized topographical data used to define the triangulated manifold. We still need an algorithm that obtains the optimal path over the triangulated manifold. This is achieved by adopting the FMM, a continuous version of the Dijkstra shortest path algorithm. Different from the "discrete" Dijkstra's algorithm which imposes the path to travel exclusively on the edges of triangles in $\mathbb{M}$, this "continuous" version of Dijkstra's algorithm enables the path to traverse through the interiors of triangles to find better solutions. The FMM is known to be optimal for solving the Eikonal equation (and therefore Problem 2) as the grid size of the triangulated manifold approaches zero (Sethian, 1999b).

In the following, we provide the detailed mathematical formulation of the link path planning problem and then introduce our methodology.

Based on the models of landforms, construction cost, and the potential required repairs, our multi-objective optimization problem of minimizing the construction cost and the total number of repairs is as follows,

(Problem 1)

$$
\begin{aligned}
& \min _{\gamma} \Phi(\gamma)=(\mathbb{H}(\gamma), \mathbb{G}(\gamma)), \\
& \text { s.t. } \gamma(A)=X_{A}, \gamma(B)=X_{B} .
\end{aligned}
$$

To calculate the construction cost and the total number of repairs of the link $\gamma$, we parametrize the curve $\gamma$ as functions of arc length, $s$; that is, every point $X \in \gamma$ can be represented by arc length $s$ as $X=X(s)$. Such a parametrization is also known as the natural definition of a curve (Burago et al., 2001). Then the construction cost and total number of repairs of the link $\gamma$ are rewritten as,

$$
\mathbb{H}(\gamma)=\int_{0}^{l(\gamma)} h(X(s)) d s, \mathbb{G}(\gamma)=\int_{0}^{l(\gamma)} g(X(s)) d s,
$$

where $h(X(s)), g(X(s)) \in \mathbb{R}_{+}^{1}$ are the construction cost and the repair rate at location $X(s)$, respectively, and $l(\gamma)$ is the length of the link $\gamma$.

This problem can be formulated as a multi-objective variational optimization problem for which calculus of variations approaches are applicable. In the following, we describe the path planning methodology we use to solve Prob- lem 1.

Since Problem 1 has multiple objectives, in general it is impossible to simultaneously optimize both the construction cost and the total number of repairs. Therefore, Pareto optimal solutions are sought. A standard method to solve Problem 1 is to formulate it as a single-objective function optimization problem through the weighted sum method, i.e.,

$$
\begin{aligned}
\text { (Problem 2) } & \min _{\gamma} \Phi(\gamma)=\int_{0}^{l(\gamma)} f(X(s)) d s, \\
& \text { s.t. } X(0)=X_{A}, X(l(\gamma))=X_{B},
\end{aligned}
$$

where $f(X(s))=c \cdot h(X(s))+g(X(s))$ and $c \in \mathbb{R}_{+}^{1}$. We call $f(X(s))$ the weighted cost. By the theory of multi-objective variational optimization (Bector and Husain, 1992; Smale, 2000), if $\gamma^{*}$ is an optimal solution for Problem 2, then it is Pareto optimal for the construction cost $\mathbb{H}$ and the total number of repairs $\mathbb{G}$. With different weights $c$ in Problem 2, distinct Pareto optimal solutions are produced.

In consequence of the formulation of Problem 2 as a single objective variational problem, the solution paths that minimize the integral are the minimum weighted cost paths. We emphasize here that Problem 2 is a continuous problem. FMM, a consistent and computational efficient numerical algorithm proposed by Sethian (Sethian, 1999b, 1996; Kimmel and Sethian, 1998; Sethian, 1999a), for solving the Eikonal equation, is adopted here to solve Problem 2 in a continuous space. Comparing with discrete optimization methods such as the Dijkstra algorithm that compute the weighted cost on a discretized grid of the region of interest, on the one hand, FMM can be proved to converge to the continuous physical (viscosity) solution as the grid step size tends to zero, i.e., achieving global optimality. On the other hand, FMM has the same computational complexity as Dijkstra algorithm, which is $O(N \log N)$, where $N$ is the total number of discretized grid points of $\mathbb{M}$ (Sethian, 1999b).

For a PDE, a solution is required to be continuous and differentiable over the entire domain according to the classical definition. Continuous physical (viscosity) solutions are weak solutions that generalize the classical definition means that they are not necessarily everywhere differentiable. These appear to be meaningful solutions to PDEs representing physical problems (Bardi and Capuzzo-Dolcetta, 2008). For the mathematical definition of the viscosity solution, it is referred to Crandall and Lions (1983).

\subsection{Derivation of the Eikonal equation}

The first step in applying FMM is to transform the variational Problem 2 to the Eikonal equation. For any point $S \in \mathbb{M}$, a cost function $\phi(S)$ that represents the minimal cumulative

This article is protected by copyright. All rights reserved. 
weighted cost to travel from End Point $B$ of the link to point $S$ is defined as,

$$
\phi(S)=\min _{\beta} \int_{0}^{l(\beta)} f(X(s)) d s,
$$

where $\beta \in \operatorname{Lip}([0,+\infty) ; \mathbb{M})$ is a Lipschitz continuous path parameterized by its length, $\left\|\beta^{\prime}(s)\right\|=\left\|\frac{d \beta(s)}{d s}\right\|=1, X(0)=$ $X_{B}$, and $X(l(\beta))=X_{S}$. By Equation (4) and the definition of $f$, and applying the fundamental theorem of the Calculus of Variations, Kimmel and Sethian (2001) have shown that $\phi(S)$ is the viscosity solution of the following Eikonal equation,

$$
\|\nabla \phi(S)\|=f(S)=c \cdot h(S)+g(S), \phi(B)=0,
$$

where $\nabla$ is the gradient operator and $\|\cdot\|$ is the 2-norm. For any point $S, \phi(S)$ is called the level set function; that is, $\{S \in \mathbb{M}: \phi(S)=a\}$ is a curve composed of all the points that can be reached from point $B$ with minimal cost equal to a. The optimal path(s) is (are) along the gradient of $\phi(S)$; i.e., orthogonal to the level curves. More precisely, we can construct the optimal path(s) by tracking backwards from $S$ to $B$, solving the following ordinary differential equation (Sethian, 1999b)

$$
\frac{d X(s)}{d s}=-\nabla \phi, \quad \text { given } X(0)=X_{S}
$$

until point $S$, is reached, where $X \in \mathbb{M}$. That is, the optimal path(s) from $A$ to $B$ for Problem 2 is(are) obtained by setting $S=A$ and then using a gradient descent of the level set function $\phi$. A finite-difference approximation, for example the first-order Euler method (Peyré et al., 2010) or a second order Heun's integration method (Sethian, 1999b) can be used to compute (6) approximately. The former is adopted in this paper. For details, the reader can refer to Peyré et al. (2010).

Note that Problem 2 may have multiple solutions. All the optimal solutions are Pareto optimal for the same construction cost $\mathbb{H}$ and the total number of repairs $\mathbb{G}$. However, they represent different paths on the manifold. The designer may select one path by his/her preferences considering other factors beyond the construction cost and the total number of repairs.

\subsection{The update scheme}

The partial differential Eikonal equation cannot be solved analytically for an arbitrary non-negative weighted cost function $f$. In fact, its solution does not necessarily need to be differentiable. Therefore, we adopt a numerical method to solve the Eikonal equation.

In Section 4, we have approximated landforms by a complete two dimensional triangulated manifolds, deriving a discretized grid model of the region under consideration. Ac- cordingly, an update scheme to calculate the value of $\phi$ at each grid point is required. In Kimmel and Sethian (1998), to compute a geodesic path on triangulated manifolds, Sethian proposes a monotone update procedure on a triangulated mesh to approximate the gradient in (5), from which the viscosity solution is obtained. The resulting path converges to the exact minimum weighted cost path as the triangulation is refined. Here, we apply it to Equation (5).

For acute triangles of the triangulated landform manifolds, the update procedure is as follows. We aim first to update the value of a center vertex, such as vertex $V$ shown in Fig. 2, which is the intersection point of several triangles. For each of these triangles, for example the triangle $\triangle V V_{1} V_{2}$ (assuming $\bar{\phi}\left(V_{2}\right)>\bar{\phi}\left(V_{1}\right)$ without loss of generality) in Fig. 2, the solutions of the following quadratic equation derived by representing Equation (5) are calculated (Kimmel and Sethian, 1998).

$$
a^{\prime} \varphi^{2}+b^{\prime} \varphi+c^{\prime}=0
$$

where

$$
\begin{aligned}
\varphi & =\phi(V)-\bar{\phi}\left(V_{1}\right), \\
a^{\prime} & =\left(a^{2}+b^{2}-2 a b \cos \theta\right) \\
b^{\prime} & =2 b\left(\bar{\phi}\left(V_{2}\right)-\bar{\phi}\left(V_{1}\right)\right)(a \cos \theta-b), \\
c^{\prime} & =b^{2}\left(\left(\bar{\phi}\left(V_{2}\right)-\bar{\phi}\left(V_{1}\right)\right)^{2}-f^{2}(V) a^{2} \sin ^{2} \theta\right) .
\end{aligned}
$$

For detailed derivation of Equation (7), the reader can refer to Kimmel and Sethian (1998). The triangle $\triangle V V_{1} V_{2}$ is used to update the value of $\bar{\phi}$ for vertex $V$ as follows. If $\varphi>$ $\bar{\phi}\left(V_{2}\right)-\bar{\phi}\left(V_{1}\right)$ and

$$
a \cos \theta<\frac{b\left(\varphi-\left(\bar{\phi}\left(V_{2}\right)-\bar{\phi}\left(V_{1}\right)\right)\right)}{\varphi}<\frac{a}{\cos \theta},
$$

that is, the solution $\varphi$ is updated from within the triangle $\triangle V V_{1} V_{2}$, then

$$
\bar{\phi}(V)=\min \left\{\bar{\phi}(V), \varphi+\bar{\phi}\left(V_{1}\right)\right\}
$$

which means that the value for vertex $V$ is updated by taking the minima of the current value $\bar{\phi}(V)$ and $\varphi+\bar{\phi}\left(V_{1}\right)$. Otherwise, $\varphi$ is not updated from within the triangle $\triangle V V_{1} V_{2}$. Only vertex $V_{1}$ or vertex $V_{2}$ is used to update the value for vertex $V$, in which case Equation (5) is rewritten as $\frac{\phi(V)-\bar{\phi}\left(V_{1}\right)}{b}=f(V)$ or $\frac{\phi(V)-\bar{\phi}\left(V_{2}\right)}{a}=f(V)$. Thus, the value for vertex $V$ is updated by the following equation.

$$
\bar{\phi}(V)=\min \left\{\bar{\phi}(V), b f(V)+\bar{\phi}\left(V_{1}\right), a f(V)+\bar{\phi}\left(V_{2}\right)\right\},
$$

In other words, the value for vertex $V$ is updated by taking the minima of the current value $\bar{\phi}(V), b f(V)+\bar{\phi}\left(V_{1}\right)$, and $a f(V)+\bar{\phi}\left(V_{2}\right)$. Since each triangle containing the vertex $V$ can produce a possible update value $\bar{\phi}$ for that vertex, in order to meet the upwind criterion (Sethian, 1999b), the smallest

This article is protected by copyright. All rights reserved. 
new value $\bar{\phi}$ for $V$ is chosen.

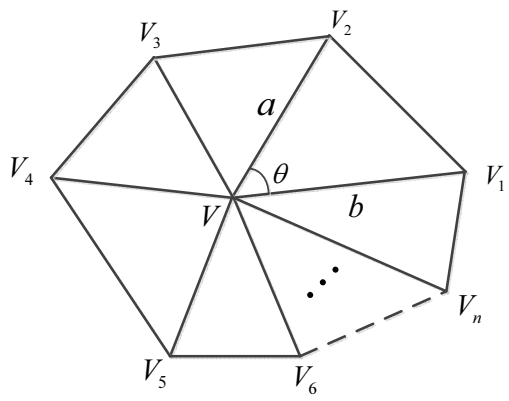

Figure 2: Illustration of acute triangulation around center vertex on landform manifolds.

For the update procedure described above, an acute triangulation is required, because the values of both vertex $V_{1}$ and vertex $V_{2}$ are needed to update the value of vertex $V$ simultaneously. Although there is a guarantee for the existence of acute triangulations for a general polyhedral surface (Saraf, 2009), no polynomial algorithm for constructing such triangulations has been found. For a given specific initially triangulated landform manifold with non-acute triangles, we may split every obtuse triangle into acute ones. If not, an "unfolding" step is necessary for the remaining obtuse angles (Kimmel and Sethian, 1998). A refinement for the splitting of obtuse angles is provided in Xin and Wang (2007). Details can be found in Kimmel and Sethian (1998); Sethian (1999a); Xin and Wang (2007).

\subsection{Algorithm for path planning}

Given the update scheme described above, and the initial value $\phi(B)=0$, the next step is to calculate the value of $\phi$ at each point $S$ on the triangulated grid of $\mathbb{M}$. From the non-negativity of $f$ and the upwind difference structure, it is useful to imagine $\phi$ as a wave function, and note that the wave propagates "one way"; that is, from $B$ outwards, and the value of $\phi$ at any point depends only on adjacent vertices having smaller values. Based on these observations, Sethian (1996) proposed to update the values of the grid points in a way similar to the Dijkstra shortest path algorithm, and named it the FMM. The algorithm is described as follows:

1. Initialization. All end points (e.g., B) are tagged as Frozen. Their nearest neighbors (one grid point away) are then tagged as Near and the value of these nearest neighbors are updated by solving (7) using Frozen points. The remaining grid points are tagged as Far;

2. The point with minimum value $\phi$ among all points with the tag Near is retagged to Frozen. If there are no such points, the algorithm is terminated. If there is exactly one such point, return to Step 3. If there are more than one such point, pick one arbitrarily and return to Step 3;

3. Find the nearest neighbors that are either Far or Near of the Frozen point found in Step 2, and update their values by solving Equation (7) using the Frozen points and change their tag to Near if they are Far;

\section{Go back to Step 2.}

Based on the above procedure, the status of points can only change from Far to Near or from Near to Frozen. The tags of points cannot be changed in an opposite direction, i.e., from Near to Far or from Frozen to Near. In Step 3, each updated point is assigned a new value that is less than its previous value. If the point is Far, it is tagged Near. In Step 2, the tag of one and only one point is changed in each loop. Therefore, the FMM is a one-pass algorithm; it does not have to "go back and correct an accepted value (of a Frozen point)". Since we can locate the grid point with minimum value $\phi$ among all points with tag Close (in Step 2) using a heap algorithm with time complexity $O(N)$, it is easily seen that FMM can be implemented with time complexity $O(N \log N)$ if $N$ is the total number of points in the grid (Sethian, 1999a).

Based on the landforms model, the construction cost model and the potential required repairs model given in Section 4 and the FMM introduced above, we provide an algorithm, called Algorithm 1, for path planning on a topographic surface. As we said at the beginning of this section, by setting different values of $c$ in Problem 2, we can obtain different Pareto optimal solutions of the construction cost and the total number of repairs. However, since FMM is a numerical method to solve the Eikonal equation (5), computational numerical errors arise in running Algorithm 1, one source of which is the approximate computation of the Eikonal equation (5) through solving Equation (7). The approximate solution has first order accuracy, i.e., the size of the error is proportional to the grid size. The other source of the numerical errors is the approximate computation of Equation (6), done here by Euler's method, and this again has first order accuracy. For more accurate, higher order approximation, the reader can refer to Sethian (1999b). Because of these numerical errors, erroneous results may occur, which can be identified by checking the dominance of the solutions. If one solution is dominated by other solutions, then it is not Pareto optimal and therefore be ignored. At the end, only the non-dominated results are presented to obtain the approximate Pareto front which is also called non-dominated front. Examples of such non-dominated optimal solutions will be provided in the next section.

We summarize the approach we used to solve the problem of

This article is protected by copyright. All rights reserved. 


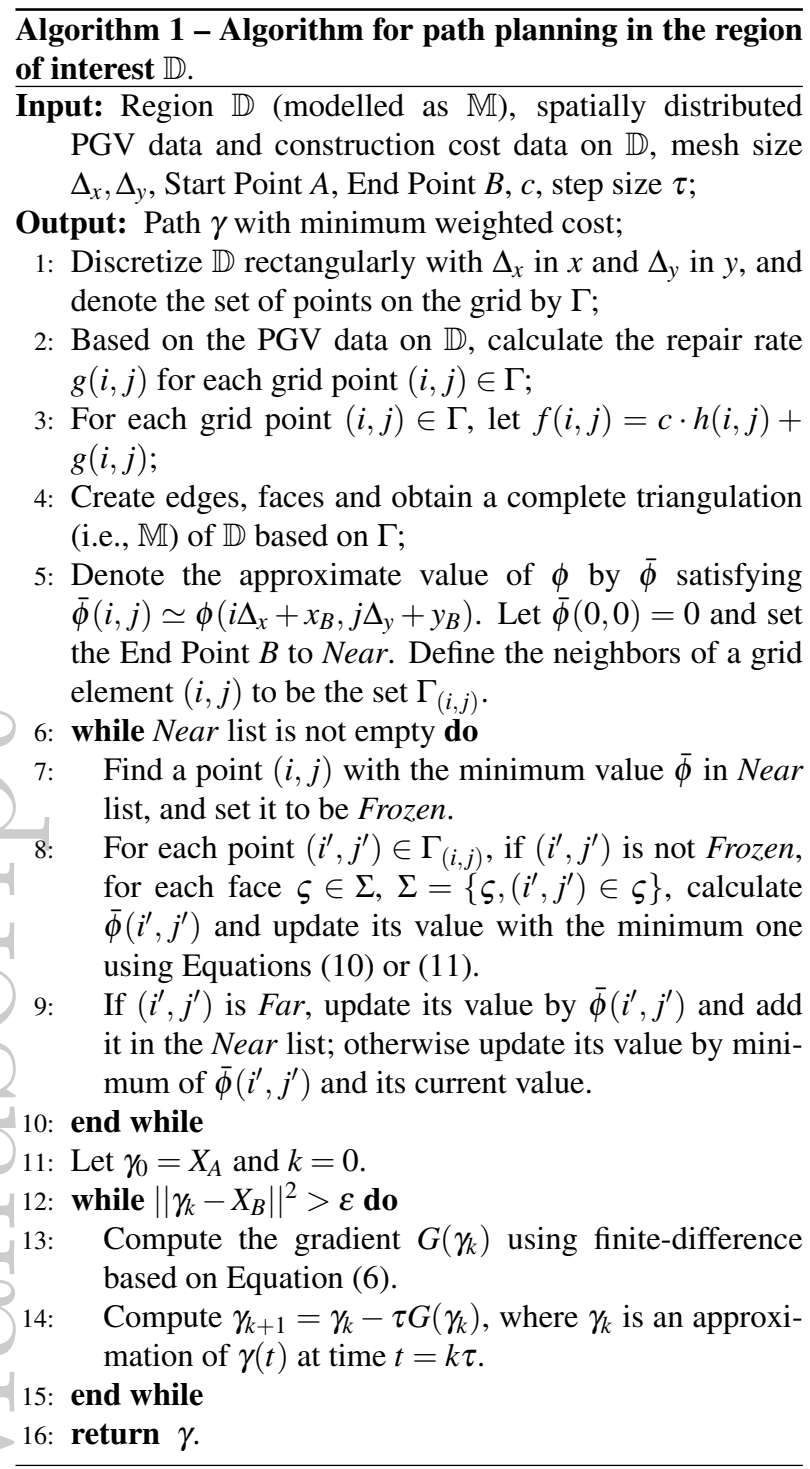

how to find the optimal path of a link to connect two points across an earthquake prone area in Fig. 3.

We emphasize that, in general, Problem 2, being a continuous problem, does not have an analytical solution except that the integrand $f(X(s))$ is very special. As we above mentioned, Kimmel and Sethian (2001) have shown that intuitively the (continuous) solution(s) of Problem 2 (i.e., path(s)) is (are) perpendicular to the solution of the (continuous) Eikonal equation (5). Again, in general, we cannot derive the analytical solution of Eikonal equation (5). Therefore, we have to resort to numerical methods. FMM, which has been proved to be a consistent and computational efficient numerical method, is used to solve Eikonal equation (5) in this paper. The (discrete) approximate solution derived by FMM converges to the continuous physical (viscosity) solution of Eikonal equation (5) as the grid step size of the topographic data tends to zero (Kimmel and Sethian, 1998). As a result, the (discrete) approximate solutions (i.e., paths)

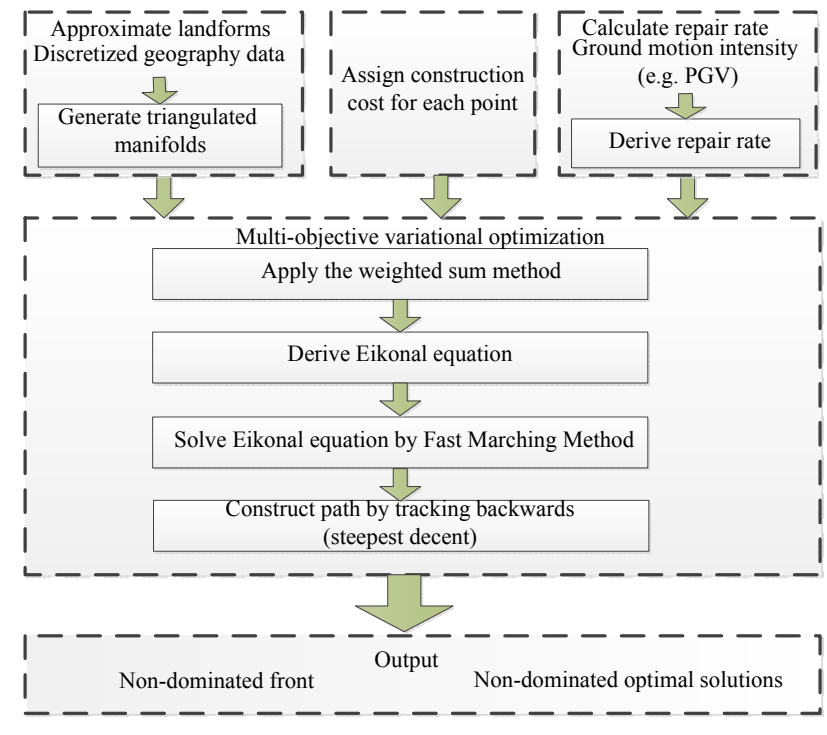

Figure 3: Framework of our approach.

converge to the (continuous) solutions of Problem 2 as well when the grid step size approaches to zero. Nowadays, high resolution (30 m or $90 \mathrm{~m}$ ) topographic data are freely available online. In future, with technological advances, and the consequent reduction in grid step size, our approach will provide increasingly accurate solutions.

\section{APPLICATIONS}

In this section, we illustrate the applications of Algorithm 1 to scenarios based on 2D and 3D landforms. We start with a simple case of $2 \mathrm{D}$ topography, where the PGV data is obtained by simulations by the Probabilistic Seismic Hazard Analysis (PSHA) method (Baker, 2008). Then, we apply the algorithm to two scenarios of 3D landforms based on earthquake hazard assessment data from USGS. Let $\delta$ (dollars $/ \mathrm{km}$ ) be the construction cost per km. We assume $\delta=1$ in the following examples. We acknowledge that the construction cost of a link is more than $\$ 1$ per $\mathrm{km}$, but if we normalize all the costs to unit cost per $\mathrm{km}$, then the path we obtain will be the correct path. The construction cost and the total number of repairs of different links are estimated. We take advantage of the low complexity of FMM, and generate many runs for different $c$ values to obtain the set of non-dominated optimal solutions.

\subsection{Application of the algorithm to a 2D land- form}

We generate PGV data based on PSHA for a simple example in which the path of the link is planned on a planar (2D) region. A single line source (e.g. a fault line 
where earthquake epicenter may occur) of earthquakes of length $20 \mathrm{~km}$ located in a 2D landform region [0,100 km] $\times[0,100 \mathrm{~km}]$ is shown in Fig. 6(a). The coordinates of the two end points $\left(x_{E}, y_{E}\right)$ and $\left(x_{F}, y_{F}\right)$ of the line source are $(50 \mathrm{~km}, 40 \mathrm{~km})$ and $(50 \mathrm{~km}, 60 \mathrm{~km})$, respectively. Following the bounded Gutenberg-Richter model (Baker, 2008), this source produces earthquakes of magnitude between $m_{\min }=5$ and $m_{\max }=6.5$ with the probability density function (PDF)

$$
f_{M}(m)=\frac{b \cdot 2.3026 \cdot 10^{-b\left(m-m_{\min }\right)}}{1-10^{-b\left(m_{\max }-m_{\min }\right)}}, m \in\left[m_{\min }, m_{\max }\right]
$$

Note that the magnitude $m$ is dimensionless (as it is given in Richter scale). We assume $b=1$ and show the PDF (12) in Fig. 4. We also assume that the spatial distribution of

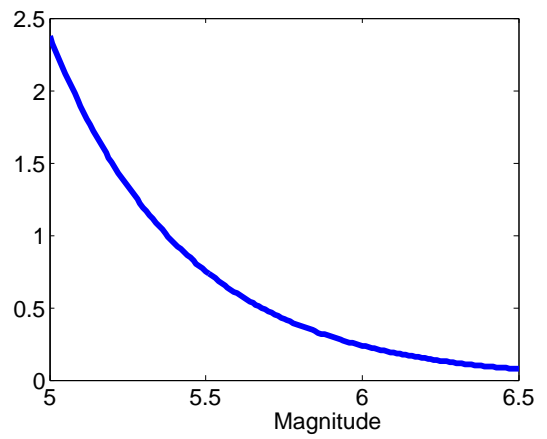

Figure 4: PDF (12) for the case $b=1$.

the epicenter of earthquakes is uniform along the line source. The geometry of the source as shown in Fig. 5 yields that the cumulative distribution function (CDF) of the epicenter distance $r$ from a site $(x, y)$ to the source is (Kramer, 1996; Baker, 2008),

$$
F_{R}(r)=P(R \leq r)=\frac{r^{\prime}}{y_{F}-y_{E}},
$$

where $r^{\prime}$ is the length of the source within distance $r$ and $y_{F}-y_{E}$ is the length of the source. Specifically, when $y<$ $y_{E}$, for example, shown by the site with circle (red color) in Fig. 5, if $d_{E} \leq r \leq d_{F}$, then $r^{\prime}=\sqrt{r^{2}-\left(x-x_{E}\right)^{2}}-\left(y_{E}-y\right)$, where $d_{E}$ and $d_{F}$ are the distances from the site to $E$ and $F$, respectively. Otherwise, $F_{R}(r)=0$ if $r<d_{E}$ and $F_{R}(r)=1$ if $r>d_{F}$. The CDF of $r$ when $y_{E} \leq y \leq y_{F}$ and $y>y_{F}$ can be derived in a similar way. The complete CDF of $r$ is as follows.

$$
F_{R}(r)= \begin{cases}y_{1}, & y<y_{E}, d_{E} \leq r \leq d_{F} \\ y_{2}, & y_{E} \leq y \leq y_{F},\left|x-x_{E}\right| \leq r \leq d_{\max } \\ y_{3}, & y>y_{F}, d_{F} \leq r \leq d_{E},\end{cases}
$$

where $y_{1}=\frac{r_{E}-\left(y_{E}-y\right)}{y_{F}-y_{E}}, \quad y_{2}=\frac{\min \left(y_{F}, y+r_{F}\right)-\max \left(y_{E}, y-r_{E}\right)}{y_{F}-y_{E}}$, $y_{3}=\frac{r_{F}-\left(y-y_{F}\right)}{y_{F}-y_{E}}, \quad d_{E}=\sqrt{\left(x-x_{E}\right)^{2}+\left(y-y_{E}\right)^{2}}$,

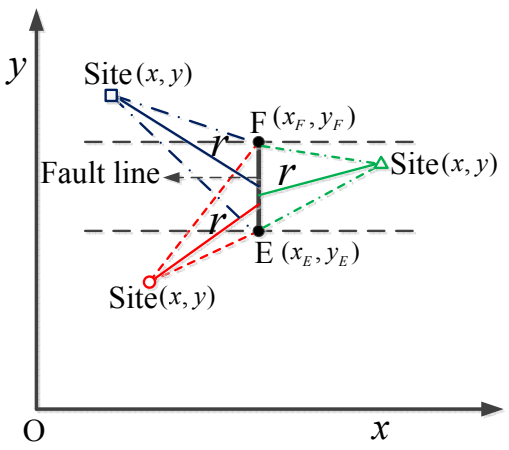

Figure 5: Illustration of derivation of the CDF in Equation (14).

$d_{F}=\sqrt{\left(x-x_{F}\right)^{2}+\left(y-y_{F}\right)^{2}}, \quad r_{E}=\sqrt{r^{2}-\left(x-x_{E}\right)^{2}}$, $r_{F}=\sqrt{r^{2}-\left(x-x_{F}\right)^{2}}$ and $d_{\max }=\max \left(d_{E}, d_{F}\right)$. The PDF $f_{R}(r)$ for $r$ is obtained by taking the derivative of the above CDF.

Given potential earthquake magnitudes and locations, ground motion measures, such as PGV, have been observed to be well-represented by a $\log$-normal distribution, i.e., $\ln v \sim$ $\mathscr{N}\left(\mu(m, r), \sigma^{2}\right)$, where $\mu(m, r)$ and $\sigma$ are the mean and standard deviation of $\ln v$ given by the attenuation relationship (Baker, 2008). In this case, we use the following dimensionally homogeneous version of the empirical formula in Cornell et al. (1979) for the mean of $\ln v$

$$
\mu(m, r)=\ln \frac{\mu^{\prime}(m, r)}{a_{1}}=2.11+1.07 m-1.55 \ln \frac{r+a_{2}}{a_{3}},
$$

where $a_{1}=1 \mathrm{~cm} / \mathrm{s}, a_{2}=25 \mathrm{~km}$ and $a_{3}=1 \mathrm{~km}$. For how to rewrite dimensionally non-homogeneous empirical formulas as homogeneous ones, the reader can refer to Sonin (2001) and Castillo et al. (2014). Note that the original version of Equation (15) in Cornell et al. (1979) has been widely used in various applications (e.g., Arango (1996), Baker (2008), Douglas (2012)).

We let $\sigma=0.64$. By Law of Total Probability, the PDF of PGV for a site $(x, y)$ is

$$
p\left(v_{x, y}\right)=\int_{M} \int_{R} p\left(v_{x, y} \mid m, r\right) f_{M}(m) f_{R}(r) d m d r
$$

and then the mean PGV is $\bar{v}_{x, y}=\int_{0}^{+\infty} v_{x, y} p\left(v_{x, y}\right) d v$. Note that the magnitude $m$ and the epicenter distance $r$ in the PSHA framework are usually assumed independent (Cornell, 1968; Petersen et al., 2014). Since there is no analytical expression for $\bar{v}_{x, y}$, we numerically compute the PDF of the PGV. In this computation, we quantize the PGV, magnitude and distance into equal bins with sizes $0.1 \mathrm{~cm} / \mathrm{s}, 0.1$ and $0.1 \mathrm{~km}$, respectively.

The shaded surface map of the mean PGV derived based on above procedure is shown in Fig. 6(a). Then the PGV is 
converted to repair rate by the following dimensionally homogeneous version of the empirical formula from Jeon and O’Rourke (2005).

$$
\ln \frac{g(x, y)}{a_{4}}=1.30 \cdot \ln \frac{\bar{v}_{x, y}}{a_{5}}-7.21,
$$

where $a_{4}=1 \mathrm{~km}^{-1}$ and $a_{5}=1 \mathrm{~cm} / \mathrm{s}$. The resulted repair rate is presented in Fig. 6(b).

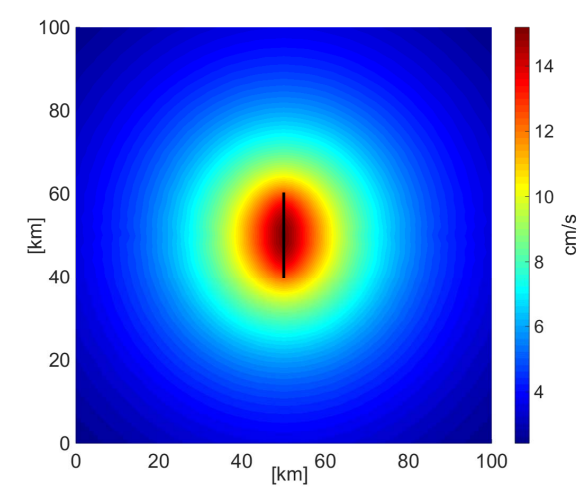

(a)

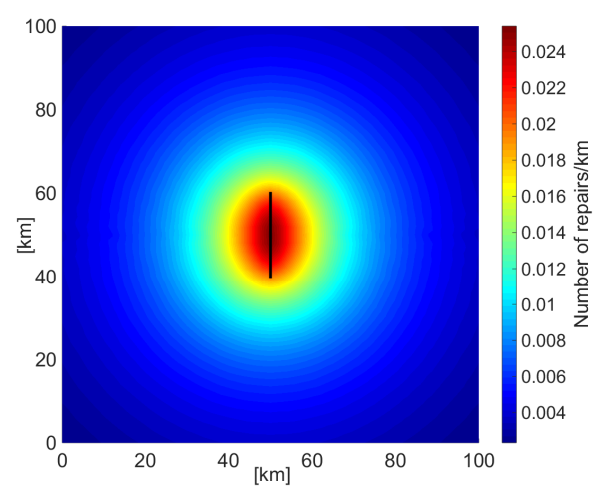

(b)

Figure 6: (a) Illustration of an example line source with length $20 \mathrm{~km}$ and the shaded surface map of mean PGV. (b) The shaded surface map of repair rate.

We plan a path of the link from the site $(10 \mathrm{~km}, 50 \mathrm{~km})$ to the site $(90 \mathrm{~km}, 50 \mathrm{~km})$. In order to see how the construction cost of the link affects the results of the path planning, we set the weight of the construction cost to different values, and then calculate the construction cost and the total number of repairs of the resulting optimal links obtained by the method given in Section 5. The results are shown in Table 2 and the corresponding paths are shown in Fig. 7(a). From Table 2 and Fig. 7(a), we can observe the trade-off between the total number of repairs and the construction cost of the link. Reducing the total number of repairs requires a higher construction cost link.

In order to derive the non-dominated front and the set of nondominated optimal solutions for the two objectives: link con- struction cost and total number of repairs, we vary the weight of the construction cost $(c)$ in the range from $10^{-4}$ to $10^{-1}$, and then calculate the optimal paths increasing $c$ by $\varepsilon$ in each path planning optimization. One can refer to Das and Dennis (1997) on how to produce a uniform distribution of points from all parts of the Pareto set. Fig. 7(b) shows the nondominated front. This non-dominated front and the corresponding non-dominated optimal solutions provide us with the results of the optimization problems of minimizing link construction cost subject to a constraint on the total number of repairs (or equivalently, on the level of survivability) and minimizing the total number of repairs (or maximizing survivability) subject to a constraint on the link construction cost.

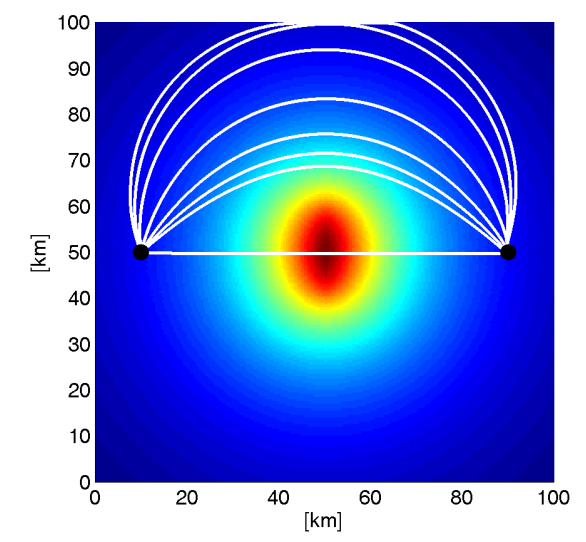

(a)

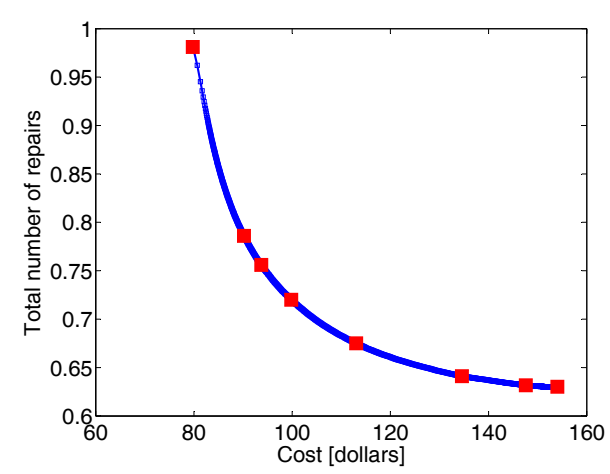

(b)

Figure 7: (a) Non-dominated optimal solutions (i.e., paths) when $c=10^{-4}, 5 \times 10^{-4}, 10^{-3}, 2.5 \times 10^{-3}, 5 \times 10^{-3}, 7.5 \times$ $10^{-3}, 10^{-2}, 2.5 \times 10^{-2}$. (b) Non-dominated front for the two objectives: (1) link construction cost, and (2) total number of repairs. The red squares are the non-dominated optimal values of the paths in (a).

To verify the ability of Algorithm 1 to avoid high construction cost areas listed in Section 4, we set up two areas, [20, $60 \mathrm{~km}] \times[40,75 \mathrm{~km}]$ and [20, $25 \mathrm{~km}] \times[40,40 \mathrm{~km}]$, which the link must avoid. For each location in these two ar- 
Table 2: Different optimal paths taking into account the construction cost and the total numbers of repairs in the case of the example line source.

\begin{tabular}{ccccccccc}
\hline$c\left(\times 10^{-3}\right)$ & 0.1 & 0.5 & 1 & 2.5 & 5 & 7.5 & 10 & 25 \\
\hline Cost (dollars) & 154.03 & 147.63 & 134.68 & 113.05 & 99.80 & 93.70 & 90.15 & 80.00 \\
Total number of repairs & 0.630 & 0.632 & 0.641 & 0.675 & 0.720 & 0.756 & 0.787 & 0.981 \\
\hline
\end{tabular}

eas, we let the construction cost function $h(x, y)=1000$. Fig. 8 shows the path of the link in the case $c=5 \times 10^{-3}$. We can see that the link successfully avoid the two high construction cost areas.

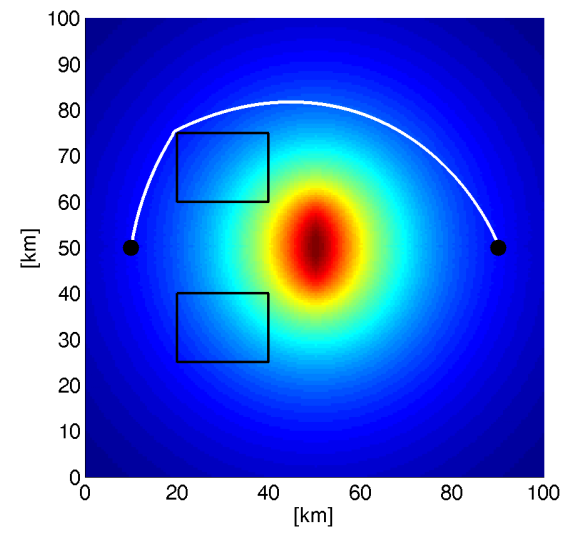

Figure 8: An illustration of avoiding high construction cost areas.

\subsection{Application of the algorithm to 3D landforms based on USGS seismic hazard map}

In the next example, we use the earthquake hazard assessment data (PGA) from USGS (http://www.usgs.gov/) that is widely applied in seismic provisions of building codes, insurance rate structure, risk assessment, and other public policy.

It contains space-delimited, rectangularly gridded data in 0.05 degree increments in longitude and latitude. We plan paths of links in two different regions $\mathbb{D}_{1}\left[40.23^{\circ},-124.30^{\circ}\right]$ $\times\left[32.60^{\circ},-114.30^{\circ}\right]$ and $\mathbb{D}_{2}\left[40.54^{\circ},-95.00^{\circ}\right] \times\left[32.75^{\circ}\right.$, $-85.50^{\circ}$ ], as shown in Fig. 9.

The land of region $\mathbb{D}_{1}$, including almost the whole California state and a large part of Nevada, is located on the west coast of US closed to the Pacific Ocean. There are several fault lines throughout $\mathbb{D}_{1}$, of which the famous San Andreas fault line is a major one. Northeast $\mathbb{D}_{1}$ is a large desert. Widely flat land of southeastern $\mathbb{D}_{1}$ is punctuated by some irregular mountain peaks such as Mt Whitney at 14,494 ft. The famous Central Valley, which is closed to the San Andreas fault line, is located in the center of $\mathbb{D}_{1}$.

Region $\mathbb{D}_{2}$ is in the US central and consists of several states such as Missouri, Illinois, Indiana, Kentucky, Tennessee, Alabama, Mississippi and Arkansas. Most of region $\mathbb{D}_{2}$ is plain, especially the central areas. A basin landform is located on the common border of Missouri, Arkansas, Mississippi and Tennessee. The New Madrid fault line is located in region $\mathbb{D}_{2}$.

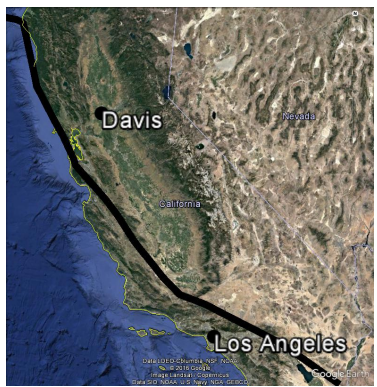

(a)

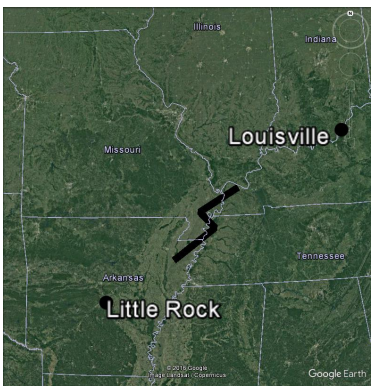

(b)
Figure 9: Geography: (a) Region $\mathbb{D}_{1}$, (b) Region $\mathbb{D}_{2}$. Black points show the cities to be connected and the black lines illustrate the fault lines. Source: Google Earth.

To calculate the repair rate of the link, we first download the PGA data (2\% probability of exceedance in 50 years, $\mathrm{Vs} 30=760 \mathrm{~m} / \mathrm{s}$ ) for each gridded geographical point and then convert it to PGV by the following dimensionally homogeneous version of the empirical equation from Wald (1999),

$$
\log _{10} \frac{v}{a_{6}}=1.0548 \cdot \log _{10} \frac{\mathrm{PGA}}{a_{7}}-1.1556,
$$

where $a_{6}=1 \mathrm{~cm} / \mathrm{s}$ and $a_{7}=1 \mathrm{~cm} / \mathrm{s}^{2}$. The shaded surface maps of PGV of the two regions are shown in Fig. 10. Then the PGV is converted to repair rate by Equation (17).

To calculate geodesic distance, we downloaded the elevation data of the corresponding areas from the National Oceanic and Atmospheric Administration (http://maps. ngdc.noaa.gov). It contains space-delimited, rectangularly gridded data with the same resolution as the repair rate data in latitude and longitude. Coordinate transformation is applied for both the repair rate data set and the geographic data to convert them from latitude and longitude coordinates to Universal Transverse Mercator coordinates. Using the landform model described in Section 4, 60, 800 faces are created for region $\mathbb{D}_{1}$ and 58,900 faces are created for region $\mathbb{D}_{2}$, and the triangulated manifold approximation of the landforms are shown in Fig. 11 and Fig. 13. 


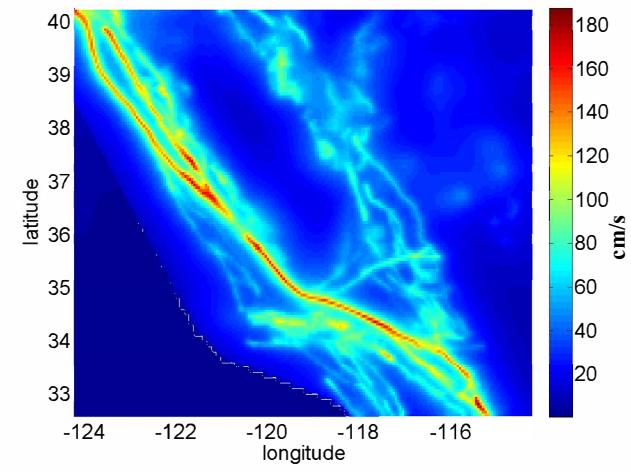

(a)

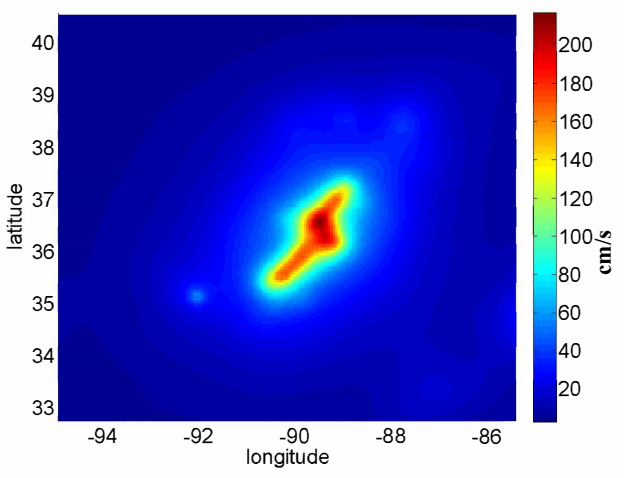

(b)

Figure 10: The shaded surface maps of the PGV (cm/s): (a) Region $\mathbb{D}_{1}$, (b) Region $\mathbb{D}_{2}$. Data is provided by USGS.

In region $\mathbb{D}_{1}$, we aim to plan the path of a link from Los Angles $\left(34.05^{\circ},-118.25^{\circ}\right)$ to Davis $\left(38.53^{\circ},-121.74^{\circ}\right)$. From Fig. 9(a) and Fig. 10(a), we can see that the link connecting the two cities should pass through the San Andreas fault line unavoidably and that it extends more than $1000 \mathrm{~km}$ through California. By letting $g(S)=0$ and $h(S)=0$ in Problem 2, we can calculate the construction cost of the minimum cost path without considering repairs and the construction cost of the path with minimum total number of repairs, which are $\$$ 598.03 and \$ 637.47, respectively. Fig. 11 shows the optimal paths when the weights of the construction cost of the link are equal to $0.0252,0.2188$ and 10. From Fig. 11, we can see that to reduce the total number of repairs through minimizing the number of points on the link that may be affected by an earthquake, the angle between the optimal link and the San Andreas fault line increases gradually around Los Angles. It then moves away from the San Andreas fault line until it approximates the one with the minimum total number of repairs. As in the previous example, we set the weight of the construction cost to be from $10^{-3}$ to 10 , and then calculate the set of non-dominated optimal solutions and the nondominated front. Fig. 12 shows the non-dominated front.

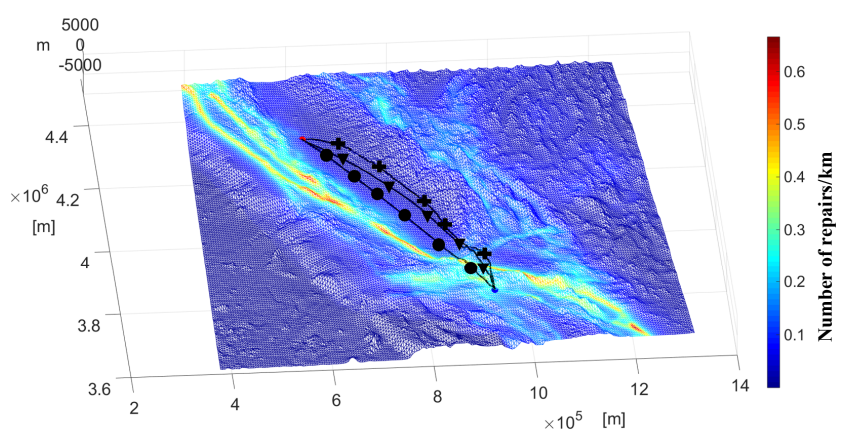

Figure 11: Illustration of triangulation in the UTM coordinate system, repair rate and non-dominated optimal solutions (i.e., paths) when $c=0.0252$ (solid circle), 0.2188 (triangle) and $c=10$ (cross). The color represents repair rate.

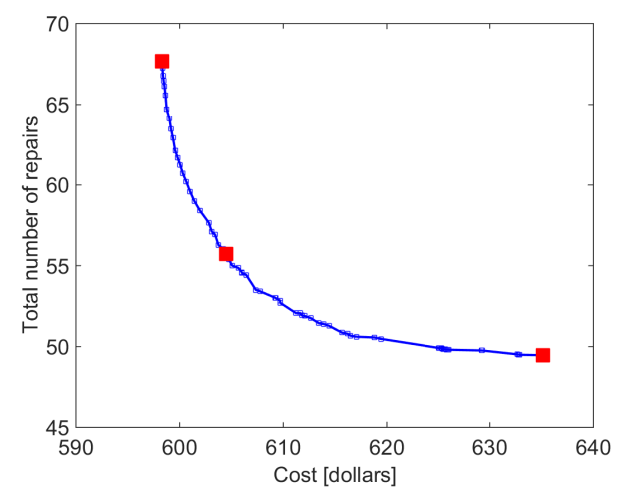

Figure 12: Non-dominated front for the two objectives: (1) link construction cost, and (2) total number of repairs. The red squares are the non-dominated optimal values of the paths in Fig. 11.

In the next and final example, we aim to plan the path of a link from Little Rock $\left(34.66^{\circ},-92.29^{\circ}\right)$ to Louisville $\left(38.24^{\circ},-85.76^{\circ}\right)$ in region $\mathbb{D}_{2}$. Since the New Madrid fault line is not as long as the San Andreas fault line, a link connecting the two cities can avoid the hazard zone if it is long enough. To calculate the non-dominated optimal solutions and the non-dominated front, we set the weight of the construction cost to be in the range between $10^{-4}$ and 10 again. Fig. 14 presents the non-dominated front. The construction cost of the minimum cost path without considering repairs and the construction cost of the path with minimum total number of repairs are $\$ 696.2$ and $\$ 1628$, respectively. Fig. 13 shows the non-dominated optimal paths when the weights of the construction cost of the link are equal to $3.8905 \times 10^{-4}, 6.6 \times 10^{-3}, 6.8 \times 10^{-3}, 0.1514$ and 10 . As in the first example, to reduce the total number of repairs, the link will be very far away from the fault line in which case the construction cost of the link will increase significantly. From Fig. 14, we can see that increasing the construction cost of the link from $\$ 700$ to $\$ 800$ can reduce the total number of 
repairs significantly (around 100 repairs). However, increasing the construction cost of the link from \$ 1000 to \$ 1600 will lead to very limited reduction in the number of repairs. This provides valuable insight to design tradeoffs between construction cost-effectiveness and survivability.

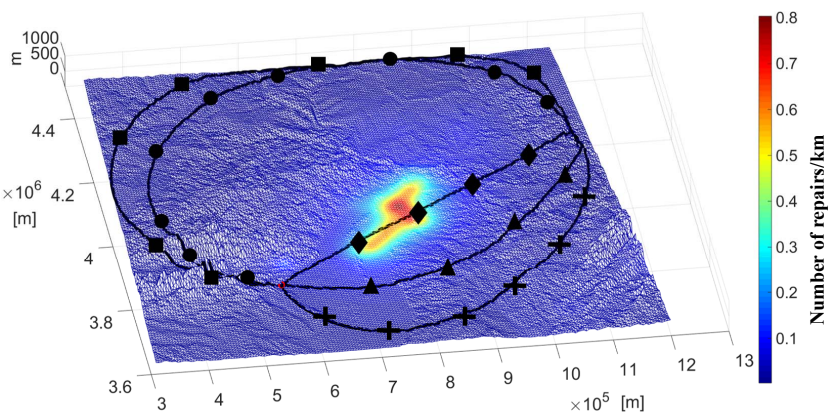

Figure 13: Illustration of triangulation in the UTM coordinate system, repair rate and non-dominated optimal solutions (i.e., paths) when $c=3.8905 \times 10^{-4}$ (diamond), $6.6 \times 10^{-3}$ (triangle), $6.8 \times 10^{-3}$ (cross), 0.1514 (solid circle) and $c=10$ (square). The color represents repair rate.

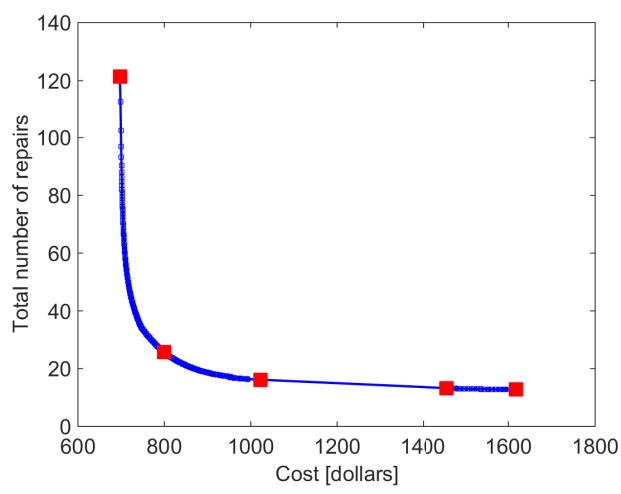

Figure 14: Non-dominated front for the two objectives: (1) link construction cost, and (2) total number of repairs. The red squares are the non-dominated optimal values of the paths in Fig. 13.

\section{CONCLUSIONS AND DISCUSSIONS}

We have developed a new method for path planning of a critical infrastructure link based on the minimization of two objectives: construction cost and potential number of required repairs. In this way, we trade-off cost and risk (especially from earthquakes) and provide the near optimal path in terms of cost for any given level of risk, and in terms of risk for any given budget. Our methodology is based on multi-objective optimization using the FMM algorithm and real data including PGV, topography, and other relevant information on the area under consideration. By a 2D landform application and two 3D landform applications, we have demonstrated that our methodology can provide a path that achieves near-minimal total number of potential repairs for a given construction cost budget, reducing both the failure probability and the time to recovery and therefore improved seismic resilience. On the other hand, given the seismic resilience required (as measured by expected number of repairs), our methodology can provide the path that achieves a cost-effective path. In this work, we did not consider other ways to improve the seismic resilience of a link by adding certain support systems, special shielding, or armored components when it passes through high risk areas. Such considerations are important, but they are beyond the scope of this paper. Nevertheless, the methodology presented in this paper can be extended to include such considerations and it is planned as future work and publications. It can also be used in a mesh network optimization for a disaster-aware design (Msongaleli et al., 2016). Other future research directions may include developing more realistic construction cost models and repair models for specific applications.

\section{ACKNOWLEDGMENT}

The work described in this paper was supported by grants from the Research Grants Council of the Hong Kong Special Administrative Region, China - Project No. CityU8/CRF/13G, and National Natural Science Foundation of China (NSFC) - Project No. 61503305. The authors would like to thank Felipe Cucker for pointing out the applicability of triangulated manifolds to the problem of link construction optimization. The authors would also like to thank the Editor and the six anonymous reviewers for their constructive comments and valuable suggestions to improve the quality of the article.

\section{References}

Adachi, T. and Ellingwood, B. R. (2009). Serviceability assessment of a municipal water system under spatially correlated seismic intensities. Computer-Aided Civil and Infrastructure Engineering, 24(4):237-248.

Adeli, H. and Wu, M. (1998). Regularization neural network for construction cost estimation. Journal of Construction Engineering and Management, 124(1):18-24.

Adeli, H. and Sarma, K. C. (2006). Cost optimization of structures: fuzzy logic, genetic algorithms, and parallel computing. John Wiley \& Sons.

This article is protected by copyright. All rights reserved. 
Alliance, A. L. (2001). Seismic fragility formulations for water systems, Part I-Guideline. http://www . americanlifelinesalliance.com/ pdf/Part_1_Guideline.pdf.

Arango, I. (1996). Magnitude scaling factors for soil liquefaction evaluations. Journal of Geotechnical Engineering, 122(11):929-936.

Baker, J. (2008). An introduction to probabilistic seismic hazard analysis (PSHA). white paper. version 1.3. Stanford University. https: //web.stanford.edu/ bakerjw/Publications/ Baker_(2008)_Intro_to_PSHA_v1_3.pdf.

Balogun, A., Matori, A., Lawal, D. U., and Chandio, I. (2012). Optimal oil pipeline route selection using GIS: Community participation in weight derivation and disaster mitigation. In Proceedings of International Conference on Future Environment and Energy 2012, volume 28, pages 100-104.

Bardi, M. and Capuzzo-Dolcetta, I. (2008). Optimal control and viscosity solutions of Hamilton-Jacobi-Bellman equations. Springer Science \& Business Media.

Bector, C. and Husain, I. (1992). Duality for multiobjective variational problems. Journal of Mathematical Analysis and Applications, 166(1):214-229.

Berry, J., King, M., and Lopez, C. (2004). A web-based application for identifying and evaluating alternative pipeline routes and corridors. In Proceedings of 2004 GITA Oil and Gas Conference, pages 1-8.

Bocchini, P. and Frangopol, D. M. (2012). Restoration of bridge networks after an earthquake: Multicriteria intervention optimization. Earthquake Spectra, 28(2):426455 .

Bruneau, M., Chang, S. E., Eguchi, R. T., Lee, G. C., O'Rourke, T. D., Reinhorn, A. M., Shinozuka, M., Tierney, K., Wallace, W. A., and von Winterfeldt, D. (2003). A framework to quantitatively assess and enhance the seismic resilience of communities. Earthquake spectra, 19(4):733-752.

Burago, D., Burago, Y., and Ivanov, S. (2001). A course in metric geometry, volume 33. American Mathematical Society Providence.

Burnett, D. R., Beckman, R., and Davenport, T. M. (2013). Submarine Cables: the handbook of Law and Policy. Martinus Nijhoff Publishers.

Cao, C. (2015). Cost effective and survivable cabling design under major disasters. $\mathrm{PhD}$ thesis, City University of Hong Kong.
Cao, C., Wang, Z., Zukerman, M., Manton, J., Bensoussan, A., and Wang, Y. (2016). Optimal cable laying across an earthquake fault line considering elliptical failures. IEEE Transactions on Reliability, 65(3):1536-1550.

Cao, C., Zukerman, M., Wu, W., Manton, J. H., and Moran, B. (2013). Survivable topology design of submarine networks. IEEE/OSA Journal of Lightware Technology, 31(5):715-730.

Carter, L., Burnett, D., Drew, S., Marle, G., Hagadorn, L., Bartlett-McNeil, D., and Irvine, N. (2009). Submarine cables and the oceans: connecting the world. UNEPWCMC Biodiversity Series No.31. ICPC/UNEP/UNEPWCMC.

Carter, L., Gavey, R., Talling, P., and Liu, J. (2014). Insights into submarine geohazards from breaks in subsea telecommunication cables. Oceanography, 27(2):58-67.

Castillo, E., Calviño, A., Nogal, M., and Lo, H. K. (2014). On the probabilistic and physical consistency of traffic random variables and models. Computer-Aided Civil and Infrastructure Engineering, 29(7):496-517.

Cavalieri, F., Franchin, P., Buriticá Cortés, J. A., and Tesfamariam, S. (2014). Models for seismic vulnerability analysis of power networks: comparative assessment. Computer-Aided Civil and Infrastructure Engineering, 29(8):590-607.

Chang, K. (2013). Introduction to Geographic Information Systems. McGraw-Hill, New York, 7th edition.

Chen, W. W., Shih, B.-J., Chen, Y.-C., Hung, J.-H., and Hwang, H. H. (2002). Seismic response of natural gas and water pipelines in the Ji-Ji earthquake. Soil Dynamics and Earthquake Engineering, 22(9):1209-1214.

Cimellaro, G. P., Villa, O., and Bruneau, M. (2014). Resilience-based design of natural gas distribution networks. Journal of Infrastructure Systems, 21(1):05014005-1-05014005-14.

Cobanli, O. (2014). Central Asian gas in Eurasian power game. Energy Policy, 68:348-370.

Cornell, C. A. (1968). Engineering seismic risk analysis. Bulletin of the Seismological Society of America, 58(5):1583-1606.

Cornell, C., Banon, H., and Shakal, A. (1979). Seismic motion and response prediction alternatives. Earthquake Engineering \& Structural Dynamics, 7(4):295-315.

Crandall, M. G. and Lions, P.-L. (1983). Viscosity solutions of Hamilton-Jacobi equations. Transactions of the American Mathematical Society, 277(1):1-42. 
Das, I. and Dennis, J. E. (1997). A closer look at drawbacks of minimizing weighted sums of objectives for Pareto set generation in multicriteria optimization problems. Structural Optimization, 14(1):63-69.

Dey, P. and Ogunlana, S. O. (1999). Decision support system for pipeline route selection. Cost Engineering, 41(10):2935 .

Douglas, J. (2012). Consistency of ground-motion predictions from the past four decades: peak ground velocity and displacement, arias intensity and relative significant duration. Bulletin of Earthquake Engineering, 10(5):13391356.

Dübendorfer, T. P. (2005). Impact analysis, early detection and mitigation of large-scale Internet attacks. $\mathrm{PhD}$ thesis, Swiss Federal Institute of Technology Zürich.

Eriksson, K., Estep, D., and Johnson, C. (2013). Applied Mathematics: Body and Soul: Volume 1: Derivatives and Geometry in IR3. Springer Science \& Business Media.

Esposito, S. (2011). Seismic risk analysis of gas distribution networks. PhD thesis, University of Naples Fegerico II.

Esposito, S., Iervolino, I., d'Onofrio, A., Santo, A., Cavalieri, F., and Franchin, P. (2015). Simulation-based seismic risk assessment of gas distribution networks. ComputerAided Civil and Infrastructure Engineering, 30(7):508523.

Florinsky, I. V. (2012). Digital terrain analysis in soil science and geology. Academic Press.

Fragiadakis, M. and Christodoulou, S. E. (2014). Seismic reliability assessment of urban water networks. Earthquake Engineering \& Structural Dynamics, 43(3):357-374.

Greenspan, D. (2000). Introduction to partial differential equations. Dover Publications, INC., Mineola, New York.

Holmes, L. and Squire, B. (2012). Least-impact right-ofway siting methodology-a route comparison application. In Proceedings of 9th International Symposium on Environmental Concerns in Rights-of-Way, Phoenix, AZ, volume 30.

Hwang, H., Chiu, Y.-H., Chen, W.-Y., and Shih, B.-J. (2004). Analysis of damage to steel gas pipelines caused by ground shaking effects during the Chi-Chi, Taiwan, earthquake. Earthquake Spectra, 20(4):1095-1110.

Iqbal, M., Sattar, F., and Nawaz, M. (2006). Planning a least cost gas pipeline route a GIS \& SDSS integration approach. In Proceedings of 2006 International Conference on Advances in Space Technologies, pages 126-130.
Jeon, S.-S. and O'Rourke, T. D. (2005). Northridge earthquake effects on pipelines and residential buildings. Bulletin of the Seismological Society of America, 95(1):294318.

Kimmel, R. and Sethian, J. (1998). Computing geodesic paths on manifolds. Proceedings of the National Academy of Sciences, 95(15):8431-8435.

Kimmel, R. and Sethian, J. (2001). Optimal algorithm for shape from shading and path planning. Journal of Mathematical Imaging and Vision, 14(3):237-244.

Kramer, S. L. (1996). Geotechnical Earthquake Engineering. Prentice Hall. Upper Saddle River, N.J.

Kobayashi, M. (2014). Experience of infrastructure damage caused by the great east Japan earthquake and countermeasures against future disasters. IEEE Communications Magazine, 52(3):23-29.

Lanzano, G., Salzano, E., de Magistris, F. S., and Fabbrocino, G. (2013). Seismic vulnerability of natural gas pipelines. Reliability Engineering \& System Safety, 117:73-80.

LaPerrière, S. (2007). Taiwan earthquake fiber cuts: a service provider view. https://www.nanog.org/meetings/ nanog39/presentations/laperriere.pdf.

Lee, J. (1991). Comparison of existing methods for building triangular irregular network, models of terrain from grid digital elevation models. International Journal of Geographical Information System, 5(3):267-285.

Liu, A. (2009). Response analysis of a submarine cable under fault movement. Earthquake Engineering and Engineering Vibration, 8(1):159-164.

Macharia, P. M. (2014). GIS analysis and spatial modelling for optimal oil pipeline route location. a case study of proposed Isiolo Nakuru pipeline route. In Proceedings of Sustainable Research and Innovation Conference, pages 9194.

Martínez, D., Velho, L., and Carvalho, P. C. (2005). Computing geodesics on triangular meshes. Computers \& Graphics, 29(5):667-675.

mi2g (2005). More than $1 \%$ GDP drop estimated per week of Internet blackout. http://www.mi2g.com/cgi/mi2g/ press/220705.php.

Miettinen, K. (1999). Nonlinear Multiobjective Optimization. Kluwer Academic Publishers, Boston.

Msongaleli, D. L., Dikbiyik, F., Zukerman, M., and Mukherjee, B. (2016). Disaster-aware submarine fiber-optic cable deployment for mesh networks. IEEE/OSA Journal of Lightwave Technology, 34(18):4293-4303.

This article is protected by copyright. All rights reserved. 
Neumann, P. J., Cohen, J. T., and Weinstein, M. C. (2014). Updating cost-effectiveness-the curious resilience of the \$ 50,000-per-QALY threshold. New England Journal of Medicine, 371(9):796-797.

Neumayer, S., Zussman, G., Cohen, R., and Modiano, E. (2011). Assessing the vulnerability of the fiber infrastructure to disasters. IEEE/ACM Transactions on Networking, 19(6):1610-1623.

Newmark, N. M. (1968). Problems in wave propagation in soil and rock. In Selected Papers By Nathan M. Newmark: Civil Engineering Classics, pages 703-722. ASCE.

O'Rourke, T. D., Toprak, S., and Sano, Y. (1998). Factors affecting water supply damage caused by the Northridge earthquake. In Proceedings of the 6th US National Conference on Earthquake Engineering, pages 1-12, Seattle, WA, USA.

Pérez, J. C., Carrillo, M. H., and Montoya-Torres, J. R. (2015). Multi-criteria approaches for urban passenger transport systems: A literature review. Annals of Operations Research, 226(1):69-87.

Petersen, M. D., Moschetti, M. P., Powers, P. M., Mueller, C. S., Haller, K. M., Frankel, A. D., Zeng, Y., Rezaeian, S., Harmsen, S. C., Boyd, O. S., et al. (2014). Documentation for the 2014 update of the United States national seismic hazard maps. Technical report, US Geological Survey.

Peucker, T. K., Fowler, R. J., Little, J. J., and Mark, D. M. (1978). The triangulated irregular network. In Amer. Soc. Photogrammetry Proceedings of Digital Terrain Models Symposium, pages 516-540.

Peyré, G., Péchaud, M., Keriven, R., Cohen, L. D., et al. (2010). Geodesic methods in computer vision and graphics. Foundations and Trends $\mathrm{R}$ in Computer Graphics and Vision, 5(3-4):197-397.

Pineda-Porras, O. and Najafi, M. (2010). Seismic damage estimation for buried pipelines: Challenges after three decades of progress. Journal of Pipeline Systems Engineering and Practice, 1(1):19-24.

Qiu, W. (2011). Submarine cables cut after Taiwan earthquake in Dec 2006. http://submarinenetworks. com/news/ cables-cut-after-taiwan-earthquake-2006.

Rose, A., Porter, K., Dash, N., Bouabid, J., Huyck, C., Whitehead, J., Shaw, D., Eguchi, R., Taylor, C., McLane, T., et al. (2007). Benefit-cost analysis of FEMA hazard mitigation grants. Natural Hazards Review, 8(4):97-111.
Røstum, J. (2000). Statistical modelling of pipe failures in water networks. PhD thesis, Norwegian University of Science and Technology.

Rui, Z., Metz, P. A., Reynolds, D. B., Chen, G., and Zhou, X. (2011). Historical pipeline construction cost analysis. International Journal of Oil, Gas and Coal Technology, 4(3):244-263.

Saraf, S. (2009). Acute and nonobtuse triangulations of polyhedral surfaces. European Journal of Combinatorics, 30(4):833-840.

Sarma, K. C. and Adeli, H. (2000b). Fuzzy discrete multicriteria cost optimization of steel structures. Journal of Structural Engineering, 126(11):1339-1347.

Schuster, R. L., editor (1991). March 5, 1987, Ecuador Earthquakes: Mass Wasting and Socioeconomic Effects. National Academies Press, Washington, D.C.

Sethian, J. (1996). A fast marching level set method for monotonically advancing fronts. Proceedings of the $\mathrm{Na}$ tional Academy of Sciences, 93(4):1591-1595.

Sethian, J. (1999a). Fast marching methods. SIAM Review, 41(2):199-235.

Sethian, J. (1999b). Level Set Methods and Fast Marching Methods: Evolving Interfaces in Computational Geometry, Fluid Mechanics, Computer Vision, and Materials Science. Cambridge Press, New York, second edition.

Sirca Jr, G. F. and Adeli, H. (2005). Cost optimization of prestressed concrete bridges. Journal of Structural Engineering, 131(3):380-388.

Smale, S. (2000). Global analysis and Economics I: Pareto optimum and a generalization of Morse theory. In The Collected Papers of Stephen Smale: Volume 1, pages 259270 .

Sonin, A. A. (2001). The physical basis of dimensional analysis. Department of Mechanical Engineering, MIT, Cambridge, MA, second edition.

Toprak, S. (1998). Earthquake effects on buried lifeline systems. $\mathrm{PhD}$ thesis, Cornell University.

Toprak, S. and Taskin, F. (2007). Estimation of earthquake damage to buried pipelines caused by ground shaking. Natural hazards, 40(1):1-24.

Wald, D. J. (1999). Relationships between peak ground acceleration, peak ground velocity, and modified Mercalli intensity in California. Earthquake Spectra, 15(3):557-564

This article is protected by copyright. All rights reserved. 
Wang, Y. and Au, S.-K. (2009). Spatial distribution of water supply reliability and critical links of water supply to crucial water consumers under an earthquake. Reliability Engineering \& System Safety, 94(2):534-541.

Wang, Y. and O'Rourke, T. D. (2008). Seismic performance evaluation of water supply systems. Multidisciplinary Center for Earthquake Engineering Research, Buffalo, NY.

Xin, S.-Q. and Wang, G.-J. (2007). Efficiently determining a locally exact shortest path on polyhedral surfaces. Computer-Aided Design, 39(12):1081-1090.

Yildirim, Y., Aydinoglu, A. C., and Yomralioglu, T. (2007). GIS based pipeline route selection by ArcGIS in Turkey. In Proceedings of the 27th Annual ESRI User Conference. pages $1-6$.

Yung, E. (2011). Project profile for South-East Asia Japan Cable System (SJC) Hong Kong segment. Technical Report 4575-OR002, Atkins China Ltd. http://www .epd.gov.hk/eia/register/profile/ latest/dir213/dir213.pdf.

Zhao, M., Chow, T . W. S., Tang, P., Wang, Z., Guo, J., and Zukerman, M. (2017). Route selection for cabling considering cost minimization and earthquake survivability via a semi-supervised probabilistic model. IEEE Transactions on Industrial Informatics, 13(2):502-511.

Zohra, H. F., Mahmouda, B., and Luc, D. (2012). Vulnerability assessment of water supply network. Energy Procedia, 18:772-783.

This article is protected by copyright. All rights reserved. 


\section{University Library}

\section{- M M N E R VA A gateway to Melbourne's research publications}

Minerva Access is the Institutional Repository of The University of Melbourne

Author/s:

Wang, Z;Wang, Q;Zukerman, M;Guo, J;Wang, Y;Wang, G;Yang, J;Moran, B

Title:

Multiobjective Path Optimization for Critical Infrastructure Links with Consideration to Seismic Resilience

Date:

2017-10-01

Citation:

Wang, Z., Wang, Q., Zukerman, M., Guo, J., Wang, Y., Wang, G., Yang, J. \& Moran, B. (2017). Multiobjective Path Optimization for Critical Infrastructure Links with Consideration to Seismic Resilience. Computer-Aided Civil and Infrastructure Engineering, 32 (10), pp.836-855. https://doi.org/10.1111/mice.12287.

Persistent Link:

http://hdl.handle.net/11343/293241 